0.021 , but this is only a rough approximation. The co-ordinates of the Observatory are$28 \cdot 6 \mathrm{~s} . \mathrm{E}$.
$52^{\circ} \mathrm{I} 2^{\prime} \mathrm{IO}^{\prime \prime} \mathrm{N}$.

A. C. Crommelin,

Trinity College, Cambridge, August ro.

\section{Macclesfield Observations.}

MANy years ago, in studying Rigaud's "Bradley," I was impressed by several references to extensive series of observations with transit and quadrant made at the observatory of Shirbourn Castle, some of which Bradley evidently thought worthy of comparison with his own inaccuracy. It has often occurred to me that these observations, if the records still exist, may well be worthy of as thorough a reduction as has been given to those of other early astronomers. Perhaps some of your readers can tell us something about these records of 1739-89.

Washington, July 30.

\section{A Lunar Rainbow.}

WET Mountain Valley in Colorado is situated some 8000 feet above the sea, and is surrounded by mountains, the Sangre de Cristo Range, on the western side, rising to some I 4,000 feet in its highest peaks. For the last few days we have had a succession of thunderstorms-dark clouds pouring forth abundant rain-which have mostly swept along the range, leaving the valley clear, and often in sunshine. Last night, at 9 p.m., there passed just such a storm, while the full moon shone brightly from the east, where it had just risen. The result was a lunar rainbow -part only of the arc, a distinct band of light, in which the several colours were hardly to be observed. The phenomenon, which was new to me and must surely be rare, lasted only about a quarter of an hour, when the storm passed on.

West Cliff, Colorado, July 25.

T. D. A. Cockereil.

\section{GLOBULAR STAR CLUSTERS.}

PHYSICAL aggregations of stars may be broadly divided into "globular" and "irregular" clusters. Although, as might have been expected, the line of demarcation between the two classes is by no means sharply drawn, each has its own marked peculiarities. We shall limit our attention, in the present article, to the first kind.

The particles of a drop of water are not in more obvious mutual dependence than the components of these objects- " the most magnificent," in the elder Herschel's opinion, "that can be seen in the heavens." Were there only one such collection in the universe, the probability of its separate organization might be reckoned "infinitely infinite"; and no less than one hundred and eleven globular clusters were enumerated by Sir John Herschel in 1864. It does not, however, follow that the systems thus constituted are of a permanent or stable character; the configuration of most of them, in fact, points to an opposite conclusion.

There may, of course, be an indefinite number of arrangements by which the dynamical equilibrium of a "ball of stars" could be secured; there is only one which the present resources of analysis enable us distinctly to conceive. This was adverted to, many years since, by Sir John Herschel. Equal revolving masses, uniformly distributed throughout a spherical space, would, he showed, be acted upon by a force varying directly as the distance from the centre. The ellipses described under its influence would then all have an identical period: whatever their eccentricities, in whatever planes they lay, in whatever direction they were traversed, each would remain invariable; and the harmony of a system, in which no perturbations could possibly arise, should remain unbroken for ever: provided only that the size of the circulating bodies, and the range of their immediate and intense attractions, were insignificant compared with the spatial intervals separating them ("Outlines of Astronomy," 9th ed., p. 636).

But this state of nice adjustment is a mere theoretical possibility. There is no sign that it has an actual existence in Nature. The stipulations, upon compliance with which its realization strictly depends, are certainly disregarded in all stellar groups with which we have any close acquaintance. The components of these are neither equal, nor equally distributed. Central compression, more marked than that due simply to the growth in depth inward of the star strata penetrated by the line of sight, is the rule in globular clusters. The beautiful white and rose-tinted one in Toucan shows three distinct stages of condensation; real crowding intensifies the "blaze" in the middle of the superb group between $\eta$ and $\zeta$ Herculis; in other cases, the presence of what might be called a nuclear mass of stars is apparent. Here, then, the "law of inverse squares" must enter into competition with the "direct" law of attraction, producing results of extraordinary intricacy, and giving rise to problems in celestial mechanics with which no calculus yet invented can pretend to grapple.

Sir John Herschel allowed the extreme difficulty of even imagining the "conditions of conservation of such a system as that of $\omega$ Centauri or 47 Toucani, \&c., without admitting repulsive forces on the one hand, or an interposed medium on the other, to keep the stars asunder" ("Cape Observations," p. I39). The establishment, however, in such aggregations of a " statical equilibrium," by means of this "interposed medium," is assuredly chimerical. The hypothesis of their rotation en blos is countenanced by no circumstance connected with them. It is decisively negatived by their irregularities of figure. These objects are far from possessing the sharp contours of bodies whirling round an axis. Their streaming edges betray a totally different mode of organization.

Globular clusters commonly present a radiated appearance in their exterior parts. They seem to throw abroad feelers into space. Medusa-like, they are covered with tentacular appendages. The great cluster in Hercules is not singular in the display of "hairy-looking, curvilinear" branches. That in Canes Venatici (M 3) has "rays running out on every side" from a central blaze, in which "several small dark holes" were disclosed by Lord Rosse's powerful reflectors (Trans. Roy. Dublin Society, vol. ii. p. 132,1880 ); showing pretty plainly that the spiral tendency, visible in the outer regions, penetrates in reality to the very heart of the system. From a wellknown cluster in Aquarius (M 2), "streams of stars branch out, taking the direction of tangents" (Lord Rosse, loc. cit. p. 162). That in Ophiuchus (M I2) has stragglers in long lines and branches, noticed by the late Lord Rosse to possess a "slightly spiral arrangement." Herschel and Baily described a remarkable group in Coma Berenices (M 53) as "a fine compressed cluster with curved appendages like the short claws of a crab running out from the main body" (Phil. Trans., vol. cxxiii. p. 458$)$.

We find it difficult to conceive the existence of "streams of stars" that are not flowing; and accordingly the persistent radial alignment of the components of clusters inevitably suggests the advance of change, whether in the direction of concentration or of diffusion. Either the tide of movement is setting inward, and the "clustering power" (to use Sir William Herschel's phrase) is still exerting itself to collect stars from surrounding space; or else a centrifugal impulse predominates, by which full-grown orbs are driven from the nursery of suns in which they were reared, to seek their separate fortunes, and lead an independent existence elsewhere. It would be a childish waste of time to attempt at present to arrive at any definite conclusion on so recondite a point; but if the appeal to "final causes" be in any degree admissible, it may be pointed out that mere blank destruction and the 
eventual collapse of the system would seem to be involved in the first supposition, while the second implies the progressive execution of majestic and profound designs.

After the lapse of some centuries, photographic measurements will perhaps help towards a decision as to whether separatist or aggregationist tendencies prevail in clusters. Allowance will, however, have to be made, in estimating their results, for the possible movements of recession or approach of the entire group relatively to the solar system, by which perspective effects of closing up or of opening out would respectively be produced.

Inequalities of brightness, to the extent of three or four magnitudes, are usually perceptible among the lustrous particles constituting these assemblages. Nor are their gradations devoid of regularity and significance. Generally, if not invariably, the smaller stars are gathered together in the middle, while the bright ones surround and overlay them on every side. Thus, the central portion of the magnificent Sagittarius cluster (M 22) accumulates the light of multitudes of excessively minute stars, and is freely sprinkled over with larger stars. The effect, which probably corresponds with the actual fact, is as if a globe of fifteenth magnitude were inclosed in a shell of eleventh magnitude stars, some of these being naturally projected upon the central aggregation. Sir John Herschel remarked of a cluster in the southern constellation of the Altar ("Gen. Cat." 4467): "The stars are of two magnitudes; the larger run out in lines like crooked radii, the smaller are massed together in and around the middle" ("Cape Observations," p. IIg). A similar structure was noted by Webb in clusters in Canes Venatici (M 3), in Libra (M 5), and in Coma Berenices (M 53) ("The Student," vol. i. p. 460). Here, again, we seem to catch a glimpse, from a different point of view, of a law connecting growth in mass and light with increase of tangential velocity-consequently, with retreat from the centre of attraction; and the assumption of an outward drift of completed suns gains some degree of plausibility.

Irregularities of distribution in clusters take a form, in some instances, so enigmatical as to excite mere unspeculative wonder. At Parsonstown, in 1850 , three "dark lanes," meeting at a point considerably removed from the centre, were perceived to interrupt the brilliancy of the stellar assemblage in Hercules. They were afterwards recognized by Buffham and Webb, and are unmistakable in one (at least) of Mr. Roberts's recent photographs of that grand object. The clusters in Ophiuchus, in Canes Venatici, and in Pegasus ("G. C." 4670) are similarly tunnelled. Preconceived ideas as to the mechanism of celestial systems are utterly confounded by appearances not easily reconcilable, so far as we can see, with the prosecution of any orderly scheme of circulatory movement. Even if absolutely vacant, the extensive clearings indicated by the phenomenon of dusky rifts, must of course, in globular clusters, be partially obliterated by the interposed light of the surrounding star-layers. They can hence become perceptible only when their development is most fully pronounced ; and, in a less marked shape, may exist in many clusters in which they defy detection.

The apparent diameter of the cluster in Hercules, in cluding most of its branches, is $8^{\prime}$; that of its truly spherical portion may be put at $5^{\prime}$. But since the sine of an angle of $5^{\prime}$ is to radius about as $\mathrm{I}: 687$, it follow's that the real diameter of this globe of stars is $I / 687$ of its distance from the earth. Assuming this distance to be such as would correspond to a parallax of $1 / 20$ of a second, we find that the more compact part of the cluster measures $558,000,000,000$ miles across. Light occupies about thirtysix days in traversing it. The average brightness of its components may be estimated at the twelfth magnitude; for, although the outlying stars are of the tenth and eleventh ranks, the central ones are, there is reason to believe, much fainter. The sum total of their light, if concentrated into one stellar point, would at any rate very little (if at all) exceed that of a third magnitude star. And one third magnitude star is equivalent to just 4000 stars of the twelfth magnitude. Hence we arrive at the conclusion that the stars in the Hercules cluster number about 4000 ; and that Sir William Herschel, in estimating them at 14,000 , erred considerably on the side of excess.

If. then, 4000 stars be supposed uniformly distributed through a sphere $558,000,000,000$ miles in diameter, an interval of $28,365,000,000$ miles, or more than ten times the distance from Neptune to the sun, separates each from its nearest neighbour. ${ }^{1}$ Under these circumstances, each must shine with about one thousand times the lustre that Sirius displays to us. Since, however, five millions of stars even of this amazing brilliancy would be needed to supply the light we receive from the sun, the general illumination of the cluster can only amount to a soft twilight, excluding, it is true, the possibility of real night on any globe situated near its centre.

At the distance conjecturally assigned to this cluster, our sun would appear as a seven and a half magnitude star ; it would shine, that is to say, about sixty-three times as brightly as an average one of the grouped objects. Each of these, accordingly, emits $1 / 63$ of the solar light; and if of the same luminosity, relative to mass, as the sun, it exercises just $1 / 500$ of the solar attractive power. The mass of the entire system of 4000 such bodies is thus equal to that of eight suns. This, however, may be regarded as a minimum estimate. The probabilities are in favour of the cluster being vastly more remote than we have here assumed it to be ; hence proportionately more massive, and composed of brighter individual bodics than results from our calculation. Differences of distance are alone adequate to account for the variety of texture observable in globular clusters. That in Aquarius, for instance, compared by Sir John Herschel to "a heap of golden sand," might very well be the somewhat coarsegrained Hercules group withdrawn as far again into space. At a still further stage of remoteness, the appearance would presumably be reached of a stellar throng in the Dolphin ("G. C." 4585), which, with low powers, might pass for a planetary nebula, but under stronger optical compulsion assumes the granulated aspect of a true cluster. And many such, their genuine nature rendered impenetrable by excessive distance, are doubtless reduced to the featureless semblance of "irresolvable" nebulæ.

But there are real as well as apparent diversities in these objects. Although smaller and more compressed clusters must, on the whole, be more remote than large, looser ones, yet "this argument," Sir William Herschel remarked, "does not exterd so far as to exclude a real difference which there may be in different clusters, not only in the size, but also in the number and arrangement of the stars." There may be globular clusters with components of the actual magnitude of Sirius; others, optically indistinguishable from them, may be aggregated out of self-luminous bodies no larger than Mars, or even than Ceres, or Pallas. There is, indeed, a strong likelihood that clusters and nebulæ form an unbroken seriesthat swarms of meteorites are connected by such interminable gradations with swarms of suns, as to admit of no impassable barrier being set up between them. ${ }^{2}$ The rifted structure, for instance, and truncated spectrum of the Hercules cluster bring it into unmistakable relations with the great nebula in Andromeda; yet it is scarcely doubtful that the one object is an assemblage of orbs exch of them, quite possibly, the rival of our sun in lustre; and the other, a collection of what we can only describe as cosmical shreds and particles. Further analogies emerge to view through the reproduction in many nebulæ of the "hairy" appendages of globular clusters, and in the spirality of arrangement characteristic of both classes

3 See J. E. Gore's similar calculation, based, however, on different dat from those assumed above. in Jocirnal Liverpool Astr. Soc. vol. v. p. róg. 2 See Mr. Lockyer's "Bakerian Lecture," p. 29 
of object. These strange and, at present, unaccountable resemblances will probably be developed and possibly be interpreted by future investigations.

\section{A. M. Clekke.}

\section{TIMBER, AND SOME OF ITS DISEASES:} XI.

T may possibly be objected that the subject of the present paper cannot properly be brought under the title of these articles, since the disease to be discussed is not a disease of timber in esse but only of timber in posse; nevertheless, while acknowledging the validity of the objection, I submit that in view of the fact that the malady to be described effects such important damage to the young plants of several of our timber-trees, and that it is a type of a somewhat large class of diseases, the slight impropriety in the wording of the general title may be overlooked.

It has long been known to forest nursery-men that, when the seedling beeches first appear above the ground, large numbers of them die off in a peculiar manner-they are frequently said to " damp off" or to "rot off." A large class of diseases of this kind is only tor familiar, in its effects, to cultivators in all parts of the world. Every gardener, probably, knows how crowded seedlings suffer, especially if kept a trifle too damp or too shaded, and I have a distinct recollection of the havoc caused by the "damping off " of young and valuable Cinchona seedlings in Ceylon.

In the vast majority of the cases examined, the " damping off " of seedlings is due to the ravages of fungi belonging to several genera of the same family as the one (Phytophthora infestans) which causes the dreaded potato disease-i.e. to the family of the Peronosporere-and since the particular species (Phytophthora omnivora) which causes the wholesale destruction of the seedlings of the beech is widely distributed, and brings disaster to many other plants; and since, moreover, it has been thoroughly examined by various observers, including De Bary, Hartig, Cohn, and others, I propose to describe it as a type of the similar forms scattered all over the world.

It should be premised that, when speaking of this disease, it is not intended to include those cases of literal damping off caused by stagnant water in ill-drained seed-beds, or those cases where insufficient light causes the long-drawn, pale seedlings to perish from want of those nutrient substances which it can only obtain, after a certain stage of germination, by means of the normal activity of its own green cotyledons or leaves, properly exposed to light, air, \&c. At the same time, it is not to be forgotten that, as conditions which favour the spread of the disease to be described, the above factors and others of equal moment have to be taken into account; which is indeed merely part of a more general statement, viz. that, to understand the cause and progress of a disease, we must learn all we can about the conditions to which the organisms are exposed, as well as the structure, \&c., of the organisms themselves.

First, a few words as to the general symptoms of the disease in question. In the seed-beds, it is often first noticeable in that patches of seedlings here and there begin to fall over, as if they had been bitten or cut where the young stem and root join, at the surface of the ground: on pulling up one of the injured seedlings, the "collar," or region common to stem and root, will be found to be blackened, and either rotten or shrivelled, according to the dampness or dryness of the surface of the soil. Sometimes the whole of the young root will be rotting off before the first true leaves have emerged from between the coty. ledons; in other cases, the "collar" only is rotten, or shrivelled, and the weight of the parts above ground

\footnotetext{
${ }^{1}$ Continued from p. 29).
}

causes them to fall prostrate on the surface of the soil ; in yet others, the lower parts of the stem of the older seedling may be blackened, and dark flecks appear on the cotyledons and young leaves, which may also be turning brown and shrivelling up (Fig. 36).

If the weather is moist-e.g. during a rainy May or June-the disease may be observed spreading rapidly from a given centre or centres, in ever-widening circles. It has also been noticed that if a moving body passes across a diseased patch into the neighbouring healthy seedlings, the disease in a few hours is observed spreading in its track. It has also been found that if seeds are again sown in the following season in a seed-bed which had previously contained many of the above diseased seedlings, the new seedlings will inevitably be killed by this"damping off." As we shall see shortly, this is because the resting spores of the fungus remain dormant in the soil after the death of the seedlings.

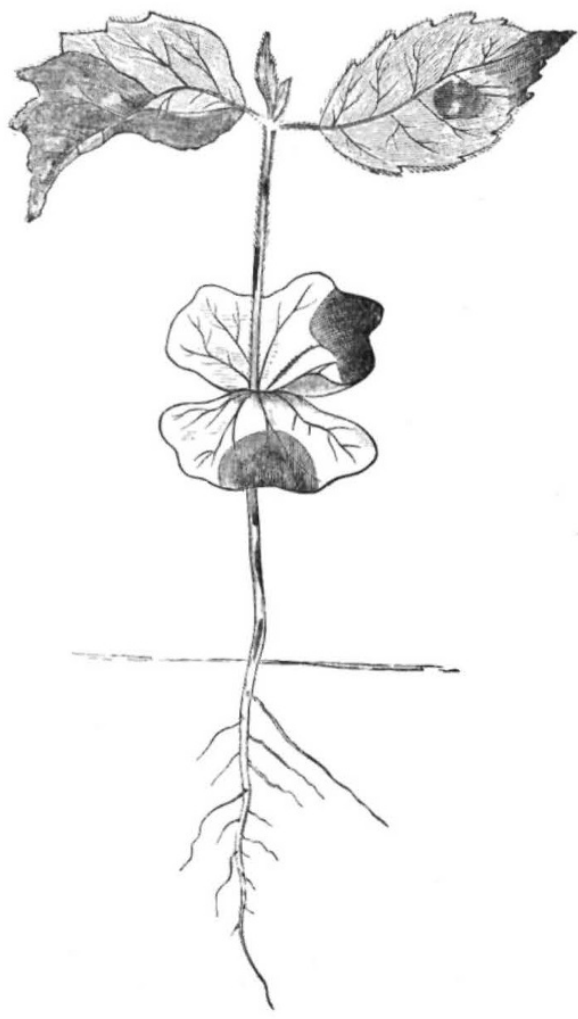

FIG. 35.-A young beech-seedling attacked by Pliytophthorra onnuzora: the moribund tissues in the brown and black patches on the young stem, cotyledon;, and leaves are a prey to the fungus, the mycelium of which is spreading from the different centre;. The horizontal line denotes the surface of the soil.

In other words, the disease is infectious, and spreads centrifugally from one diseased seedling to another, or from one crop to another: if the weather is moist and warm-" muggy," as it is often termed - such as often occurs in the cloudy day's of a wet May or June, the spread of the disease may be so rapid that every plant in the bed is infected in the course of two or three days, and the whole sowing reduced to a putrid mass; in drier seasons and soils, the spread of the infection may be slower, and only a patch here and there die off, the cliseased parts shrivelling up rather than rotting.

If a diseased beech seedling is lifted, and thin sections of the injured spots placed under the microscope, it will be found that numerous slender colourless fungus-filaments are rumning between the cells of the tissues, branching and twisting in all directions. Each of these fungus-fila- 